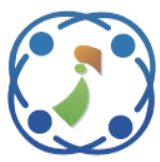

\title{
Automated Approach for Extraction of Microaneurysms and Hemorrhages in Retinal Fundus Images
}

\author{
Hussain F. Jaafar ${ }^{1 *}$ \\ Hilal Al-Libawy ${ }^{2}$ \\ Qais K. Al-Gayem² \\ ${ }^{l}$ Biomedical Engineering Department, Faculty of Engineering, University of Babylon, Babylon, Iraq \\ ${ }^{2}$ Electrical Engineering Department, Faculty of Engineering, University of Babylon, Babylon, Iraq \\ * Corresponding author's Email: hussain_engi@yahoo.com
}

\begin{abstract}
Early detection and quantification of microaneurysms and hemorrhages can potentially help reduce the risk of vision loss. In this paper, an automatic algorithm for extraction of microaneurysms and hemorrhages from color fundus images is presented. The color retinal image is analyzed and segmented using a top-down segmentation after some image enhancement steps, namely shade correction and contrast enhancement. The whole segmented images create a dataset of regions. Edge detection followed by region-growing techniques are also used to refine the top-down segmentation results. Segmented regions could be classified into true lesions and artifacts using a set of prior features like size, edge strength, color and texture. These are extracted and then utilized with a local-region properties classifier. To validate the outcomes of the proposed method, clinician's references were used to calculate the average performance measures. The proposed method achieved sensitivity of $98.8 \%$, specificity of $97.7 \%$, accuracy of $99.3 \%$, and positive predictive value of $83.7 \%$ per-pixel basis. Superior performance measures and high computation speed have assured that the proposed method is more efficient and reproducible compared to the manual methods. The significant novelty of this work is in the use of three refining operations, i.e. Edge detection, region growing and classification to achieve optimal performance especially for the sensitivity and positive predictive value. The performance measures of the proposed algorithm are compared against many recent automated systems and are found to outperform most of them. The proposed algorithm performance is very close to that of the specialist ground truth. Hence, premium performance and high computation speed of this algorithm make it promising approach for a computer-aided mass screening of retinal diseases.
\end{abstract}

Keywords: Medical image processing, Retinal fundus image, Image enhancement, Microaneurysms and hemorrhages, Top-down segmentation, Edge detection, Region growing, Image classification.

\section{Introduction}

People with high glucose rate in the blood are exposed to diabetes, and long-standing diabetes may damage thin retinal vasculature leading to diabetic retinopathy (DR). The DR is an eye disease that is associated with diabetes and considered the main cause of blindness. Treatment can slow down the progression, when DR is diagnosed in its early stages. However, early diagnosing of DR is not always easy, because it almost has faint warning signs at early stages. Earliest harms of DR are leakages in the thin retinal vessels and forming bloody lesions.
Microaneurysms (MAs) are the first popular lesions in the early DR stage, and they are considered as the common marker of the co-existent retinal oedema, which is the main reason of vision loss. The MAs may cause narrowing retinal blood vessels and weakening their walls. In addition, long-standing diabetes may cause rupturing MAs and leading to red dot hemorrhages (HEs).

Manual detection and quantification of MAs and HEs require long time and prone to errors by the observer. A computer aided extraction of MAs and HEs could accomplish accurate and fast diagnosis and help the clinician to decide the appropriate treatment. Fig. 1 illustrates retinal fundus image with different lesions including MAs and HEs. 


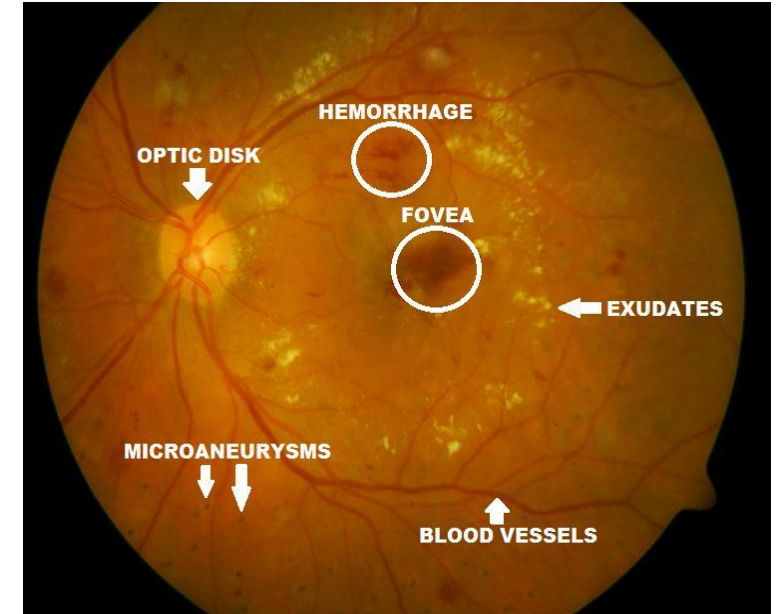

Figure. 1 Retinal image containing microaneurysms and hemorrhages [1]

Nowadays most of the DR lesion detection researchers focus on detection of MAs and HEs because they are the first clinical signs of DR. Automated detection of MAs and HEs is a big challenge due to the resemblance of their features with those of retinal structures, particularly the blood vessels and fovea. Variability of MAs and HEs features among different patients makes it difficult to assign specific set of features for the classification stage. In addition, and in case of poor contrast images, visibility of all lesions including MAs and HEs is weak and this makes precise extraction of lesions difficult task.

Many studies for extraction of MAs and HEs can be found in the literature. Niemeijer, et al., 2005 [2] proposed a red lesion detection algorithm, where pixel classification approach is used to extract red lesions and blood vessels from the background followed by blood vessels removal from the detection result leaving only red lesions. In the classification stage, a k-nearest neighbor classifier is used to separate and then reject blood vessels from the image. The main defect of this work is that the algorithm detected all positive images in the test set correctly as positive while classifying a number of negative images as positive.

Walter et al., 2007 [3] presented a new algorithm for detection and quantification of MAs. They used a diameter closing technique to extract MAs candidates and then select MAs features to separate extracted candidates into real MAs and spurious objects. This method achieved outcomes with sensitivity (SE) of $88.5 \%$. The drawback of this work is represented by the low SE and the absence of the other performance measures.

Acharya et al., 2009 [4] proposed an edge detection method followed by morphological techniques to detect MAs with overall SE of $82.4 \%$, specificity (SP) of $86.5 \%$, and positive predictive value (PPV) of $95.5 \%$. This system could identify different types of lesions with superior PPV and low SE and SP. Anyhow this defect can be remedied by more diverse data and better features.

A combination of mathematical morphology and pixel classification has been proposed by Kande et al., 2010 [5] to detect red lesions with SP of $91 \%$ and SE of $100 \%$ in terms of lesions. Junior and Welfer, 2013 [6] proposed a mathematical morphology based algorithm for automated detection of MAs and HEs. This algorithm has five stages: a preprocessing of the fundus image, enhancement of the low intensity retinal structures, detection and elimination of the blood vessels and the fovea, and achieved SE of $87.7 \%$ and SP of $92.4 \%$. Comparison Table, presented in this paper, shows dissatisfactory results especially in the SE. The reason of this is that the algorithm cannot detect small-sized MAs which are confused with noises.

A scale-space based method is proposed by Soares et al., 2014 [7] for the MAs detection. This method performs a segmentation of the retinal vasculature and defines a global set of MAs candidates using both coarser and finer scales with performance of $96.9 \% \mathrm{SE}$ in lesion based. In this paper the algorithm was tested using SE in terms of lesions while other important performance measures are not reported.

Tamilarasi and Duraiswamy 2015 [8] have developed an efficient method to automate the detection of MAs by using wavelet-based Gaussian mixture model and microstructure texture feature extraction. Bottom-hat filtering and gamma correction method are applied to the green component as a preprocessing stage to enhance the image, followed by extraction of microstructures by Gaussian profiles in wavelet domain using the threelevel generative model. A classification process to discriminate true-MAs from non-MAs was used and obtained competitive performance results with SE of $98.9 \%$, SP of 97.65 , and ACC of $98.33 \%$. But the $\mathrm{PPV}$, which is important to show percentage of the false positives in the findings, is not reported.

Seoud et al. 2015 [9] presented a new algorithm based on many stages including, image preprocessing, identification of red lesion candidates, and classification using a new set of shape features, called Dynamic Shape Features. The candidate regions are first segmented; then, color and shape features are extracted and used in the classification. This method has been tested per-lesion basis and achieved the following performance measures: SE of $92.9 \%$ and SP of 85.9. But the PPV measure and validation perpixel basis were not reported in this work. 
Ren et al. 2016 [10] proposed an ensemble framework using boosting, bagging, and random subspace to detect MAs from retinal image. A combination between ensemble and adaptive oversampling was used to overcome the problem of induction biases which are produced due to imbalance data. To enhance the algorithm performance, a classification operation was applied using extreme learning machines. The proposed method achieved poor classification performance, such as: ACC of $88.2 \%$, SE of $96.1 \%$ and SP of $82.1 \%$.

Gharaibeh 2017 [11] presented a new method for detection of MAs. This method includes the following stages: image preprocessing, detection and elimination of the blood vessels and the fovea, and feature extraction followed by classification of MAs using neural networks and fuzzy logical models. This method achieved ACC of $99 \%$, SE of $99 \%$ and SP of $96 \%$, while the PPV is not reported.

Mamilla et al. 2017 [12] proposed a coarse-tofine strategy for detection of red lesions, it is based on combination of phase congruency and mathematical morphology to extract candidates of red lesions as the coarse stage. The computation of phase congruency is achieved by using extended 2D $\log$ gabor filter. In the fine stage a support vector machine classifier is used to refine the red lesion candidates. This method was tested, per-lesion basis, and achieved the following performance measures: ACC of $99.7 \%$, SE of 99.3, and SP of $99.2 \%$, while the test in terms of pixels is not reported.

Manjaramkar and Kokare 2018 [13] proposed a new method using maximally stable extremal regions (MSER) for detecting different shapes and sizes of HEs in retinal images. This method includes three stages: (I) extraction of $\mathrm{HE}$ candidates, (II); extraction of features (III); detection of fine HEs. This method achieved SE of $94.89 \%$ and SP of $98.9 \%$ per-lesion basis, but it was not tested in terms of pixels.

Khojasteh et al. 2018 [14] proposed a new method using probabilistic output from convolution neural network to detect both bright and red lesions, namely exudates, MAs and HEs. The automated method was validated using patch and image-based analysis of retinal images. The advantage of the proposed method is the ability to detect both bright and red lesions automatically and simultaneously. This method achieved ACC of $94 \%$, SE of $85 \%$. SP of $96 \%$, and PPV of $83 \%$. The SE is low compared to the previous related works.

Pramar and Lakshmanan 2019 [15] proposed a new method to detect MAs and Exudates using Convolutional Neural Networks to extract lesion candidates and then for prediction of the model, they trained the model on a GPU. GPU-accelerated library of primitives aimed at Deep Neural Networks, NVIDIA CUDA Deep Neural Network (cuDNN) is used in their model. The advantage of this model is its ability to detect both bright and red lesions but with moderate performance measures, i.e. SE of $94 \%$ and SP of $95 \%$.

Lokuarachchi et al. 2019 [16] proposed a new method to detect red lesions using image peprocessing followed by detection of red lesion candidates, where some machine learning algorithms such as Support Vector Machines, K-nearest Neighbors, Decision Tree, and Classification Ensemble were used to separate true red lesions from artifacts with SE of $92.1 \%$ and SP of $88.7 \%$.

Zhang et al. 2019 [17] presented a new method for detection of MAs using a deep neural network with a multilayer attention mechanism. First a series of image enhancement operations are performed to improve the quality of retinal images. Then the attention mechanism, multiple feature layers with obvious target features are used to extract preliminary candidates of MAs. Finally, the spatial relationships between MAs and blood vessels were used to remove traces of blood vessels to achieve final MAs image with low SE (86.8\%) and competitive PPV (83.1\%) in terms of pixels.

In our work, the proposed method consists of four stages: first; the green channel image is preprocessed using the techniques of shade correction followed by contrast enhancement. The second stage includes the initial extraction of MAs using a top-down segmentation based on computation of local statistics of MAs and HEs candidates. In the third stage, the result of the preceding operations is refined using the edge detection and region-growing techniques followed by removing traces of blood vessels and fovea. The fourth stage presents a classification of real lesions (MAs and HEs) and removing non-lesions using a local-region properties classifier with a set of 14 features.

The proposed method is designed to be automatic and free from human intervention to extract MAs and HEs from retinal images with excellent performance and very fast computation speed compared to the recent related studies. This paper is organized as follows: Section 2 presents the material sources, used in this work, and explains details of the proposed method stages including image preprocessing, Segmentation of MAs and HEs candidates, Refining of MAs and HEs candidates, and classification. Section 3 introduces experimental results, discussion of the results and the influence of the method parameters on the results and on the performance 
measures, and comparison of the proposed method with the previous related works. Section 4 presents conclusions of the authors.

\section{Materials and methods}

In this work, a novel method has been used to detect and quantify MAs and HEs from retinal images. To train and test the proposed method, three sets of normal and abnormal retinal images from different databases have been used.

\subsection{Materials}

The databases, used in this work, include the following sources:

- Training set: It consists of 30 retinal images from the Messidor database (Walter et al., 2002 [18]) of which 21 images are affected by MAs and HEs and are manually marked by experts. They are captured at $45^{\circ}$ field of view with size of $640 \times 480$ pixels.

- Testing set: It is taken from a variety of database sources and it consists of 209 retinal images. This set is supplied by the following sources: 89 retinal images are taken from the DIARETDB1 database (Kauppi et al., 2007 [1]), of which 42 affected by MAs and HEs. 120 retinal images are taken from the DIARETDB0 database (Kauppi et al., 2006 [19]), of which 110 contain MAs and HEs. These images are acquired at $50^{\circ}$ field of view and a resolution of $1500 \times 1152$ pixels.

\subsection{Image preprocessing}

Due to differences in physical features of eye patients, most retinal images are different in visual contrast. Because of variability in the acquisition process of retinal images, most retinal images are different in illumination uniformity. These problems affect the algorithm performance for automated detection of lesions and make it difficult to separate MAs and HEs from other red features in retinal images. Hence, to achieve input images with better qualities, preprocessing steps were carried out using techniques of illumination equalization followed by adaptive contrast enhancement.

The processes of illumination equalization are significant steps to prepare images with better quality before postprocessing steps. To allow the proposed method applicable to a variety of databases irrespective of the resolution the color fundus image is initially resized to the size of the training images $(640 \times 480$ pixels $)$. To improve illumination uniformity of the image, a morphological technique, namely the top-hat operator is used. The top-hat approximation to the background of green

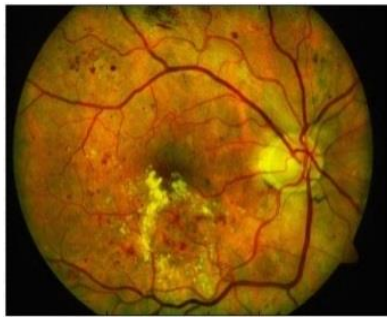

(a)

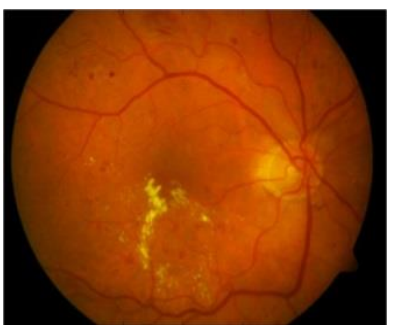

(c)

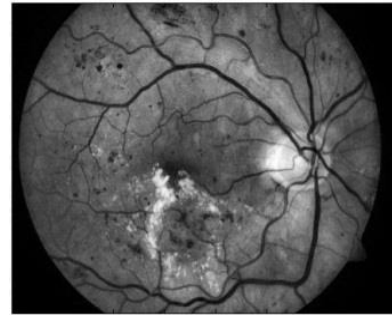

(b)

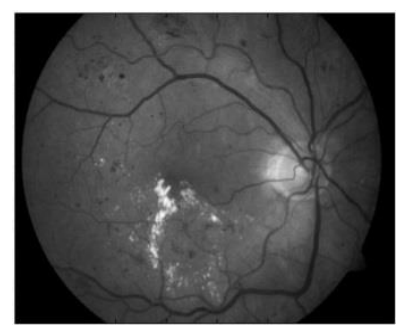

(d)
Figure. 2 Image preprocessing: (a) Original color image, (b) Green component image, (c) Preprocessed color retinal image, and (d) Preprocessed green component image

approximation to the background of green component image using mathematical morphology steps. Alternating morphological opening and closing with suitable size of structuring element and number of repetition were used to avoid entirely fitting within small candidate objects. illumination equalized image could be achieved by subtracting approximated background from the green component image.

The reason behind using alternating sequential opening and closing, in the approximation of the background is to avoid appearance of undesired objects near the optic disk and image borders. Experimental results showed that a disk-shaped structuring element with 3 pixels radius and 8 alternative repetition were suitable with the image size of $640 \times 480$ pixels. Effectiveness of the illumination equalization operation depends on the uniformity of the image as well as on the shape and size of the structuring element.

In some poorly contrasted retinal images, retinal structures and lesions are faint and not very clear, thus their segmentation from the background is not always easy task. Consequently, contrast enhancement of retinal photographs is crucial to increase visual appearance of MAs and HEs. A Contrast Limited Adaptive Histogram Equalization (CLAHE) is effective when is used with HSI color space, hence CLAHE was applied to the intensity component of HSI color space after image conversion from RGB to HSI color space. The HSI color space is more suitable because the intensity component is separated from the other two components. 
The CLAHE enhances the image contrast using intensity transformation to small regions instead of the entire image. The contrast of each small region is enhanced using histogram equalization. The resulting HSI components are converted again to RGB space to achieve the preprocessed image from the green component image. Fig. 2 (a) and Fig. 2 (b) show an original color retinal image and its green component image respectively, while Fig. 2(c) and Fig. 2(d) illustrate their preprocessed results.

\subsection{Segmentation of MAs and HEs candidates}

The MAs and HEs are mainly caused due to leakages in tiny ends of the blood vessels. Hence, they have almost spherical or elliptical shapes with different diameters. Consequently, they have different features and shapes from the other retinal structures, which have similar intensities, i.e. the blood vessels and the fovea (the centre of vision). Extraction of MAs and HEs here is mainly based on the top-down segmentation which includes two steps: Firstly, the image is segmented into smaller regions and secondly, evaluation process is applied to the segmented regions. These two operations are repeated iteratively until reaching predefined criteria to obtain candidates with similar characteristics, thus being more suitable for reliable thresholding. The proposed top-down segmentation could be implemented by applying the following steps:

1. Describe global statistics of the preprocessed image, thus being considered as the result of initial segmentation.

2. Extract regions whose local statistics, namely, the mean and the variance, are completely different from the global statistics.

3. The local statistics of all extracted subregions in the step 2 are considered as the new global statistics.

4. Steps 2 and 3 are applied to the new subregions until accomplishing the homogeneity for all created sub-regions.

To examine the homogeneity of the region $H$, the predicate $P$ is used, when it is false, the region is again sub-divided into small regions, i.e. $H_{i}, i=$ $1, \ldots, n$, where the value of $n$ depends on the comparison between local mean values, where these values are calculated inside a small sliding window, with the global mean value. The algorithm is designed to operate on different stages of partitioning as indicated in Eq. (1) and Eq.(2):

$$
H \Rightarrow\left\{\begin{array}{cc}
\cup_{i=1}^{n} H_{i} & \text { if } P(H) \text { is false } \\
H & \text { otherwise }
\end{array}\right.
$$

The predicate $P$ could be expressed as follows:

$$
\begin{aligned}
& P(H) \\
& =\left\{\begin{array}{c}
\text { true if } \max _{(x, y) \in R}[f(x, y)]-\min _{(x, y) \in R}[f(x, y)] \leq \varepsilon \\
\text { false otherwise }
\end{array}\right.
\end{aligned}
$$

where $f(x, y)$ is the gray-level of the pixel located at $(x, y)$ and $\varepsilon$ is a predefined homogeneity threshold obtained empirically. Proper selection of $\varepsilon$ is vital to avoid over or under-segmentation and to achieve a suitable balance between the performance measures and the computation complexity, as will be discussed later. In this stage, coarse MAs and HEs with other dark retinal structures, such as blood vessels and fovea, were extracted. To remove all non-lesion candidates from the results of this coarser stage, finer operations are vital. In addition, the blood vessels and fovea should be detected separately and then eliminated from the results of the refining stage.

\subsection{Refining of MAs and HEs candidates}

The resulting image from the preceding stage is sub-divided into a number of homogeneous regions to increase the reliability of subsequent MAs and HEs extraction. In the absence of any prior knowledge about their presence and distribution, global thresholding will not be the appropriate choice for lesion extraction. Therefore, the fact that MAs and HEs have distinct borders while other red and dark areas namely the other shady areas which have no such clear borders, should be exploited to discriminate real red lesions from non-lesions.

The proposed fine segmentation method is based on combination of edge detection and regiongrowing techniques. First, edges of each region are determined using the Canny edge detector, and then thresholded with a calculated threshold value $(\alpha)$. Average intensity of the green component image $\left(I_{g r n}\right)$ is used to calculate $\alpha$ automatically. Experimental outcomes have showed that the threshold $\alpha=0.09 I_{g r n}$ can achieve reasonable balance in performance measures, particularly between the main two meaningful measures, i.e. the SE and the PPV. The most MAs and HEs objects are detected with this $\alpha$, because missing of any MAs and HEs candidate will not be retrieved in later stages.

Based on hand-labelled images, annotated by experts, initial evaluation to the resulting MAs and HEs candidates showed that some of them suffer from bad representation to the actual lesions. Hence, a region-growing technique can rectify this defect by growing back lesion locations on the original image. 
For this, the original green channel image and the resulting binary image were used together in such a way that the lowest intensity in each gray-level region corresponding to the binary candidate region is used as a seed in the approach of region-growing.

The first step in the region-growing is reducing all binary candidates into many single pixels located at the center of each one to act as the seeds for the technique of region-growing. Neighboring pixel that should be joined to the grown object is assigned based on the predefined criteria and on difference between the intensities of the seed and the spatial corresponding pixel of the image background. The threshold $(\beta)$ of region-growing procedure can be calculated using the estimated background $I_{b g}$ and the intensity of the starting point, i.e. the intensity of the seed pixel $\left(I_{\text {seed }}\right)$ as in Eq. (3).

$$
\beta=I_{\text {seed }}-x\left(I_{\text {seed }}-I_{b g}\right)
$$

where $x$ is a fraction between 0 and 1 . The factor $x$ assigns how much of MAs and HEs features should be used in the classification. The greater the value of the factor $x$, the bigger areas of lesion features are segmented. Based on experimental tests with several values of $x$ between 0 and 1, it appears that $x=0.6$ can ensure reasonable balance between the most informative performance measures namely, the SE and the PPV.

Although, the proposed method can perform with competitive performance of MAs and HEs extraction, some results may contain traces of blood vessels and other non-lesion objects. The fovea is a dark area and has similar features as dark lesion, so it is sometimes detected as big HE in the middle of retinal image. To remove structural traces from resulting image, fovea mask and blood vessels image, were subtracted from the fine segmentation result, where the resulting image will include real dark lesions and few artifacts to be removed later by the classification steps.

Retinal blood vessels appear as dark lines with different diameters, lengths, and orientations. Then, the multiscale approach is suitable to isolate features of blood vessels from the background. The fovea is localized by defining a candidate region of interest with reference to the established retinal landmarks, followed by a shape and intensity search. The blood vessels and fovea detection approaches, adopted in this work, are explained in details in [20].

\subsection{Classification}

To classify MAs and HEs and then discriminate them from other non-lesions in the resulting image, a local-region properties classifier was used to separate real MAs and HEs from eccentric and big artifacts. Real MAs and HEs blobs were separated from spurious objects using grayscale features and binary features. The binary features relates to the candidate shape, such as convex area minor axis length, major axis length, solidity, and eccentricity and others. Predefined properties from relationships between these features or some limits in their values were used to discriminate real dark lesions from non-lesions.

These properties might be created due to long and narrow objects from blood vessels that had not been successfully removed (due to missing them in the blood vessels detection). Additionally, convex areas which are formed in the edges of retinal images due to some abnormal shady areas (created from shade correction operation) might be confused for MAs and HEs. A set of 14 features in both binary and grayscale representations are used for every particular dark lesion candidate as input to the local-region properties classifier. The binary and grayscale features adopted in the classification are as follows:

1. Solidity of lesion candidate $(S)$,

2. Eccentricity of lesion candidate $(E)$,

3. Area $(A)$; No. of pixels in candidate region.

4. Perimeter $(P)$; Number of pixels around candidate region.

5. Circularity $(C)$; The measure of roundness from perimeter and area $\left(C=4 \pi A / P^{2}\right)$,

6. Major axis length $(L)$,

7. Minor axis length $(W)$,

8. Aspect Ratio $(R), R=L / W$,

9. Mean intensity $\left(I_{m}\right)$ of the candidate in the green component,

10. Standard deviation $\left(S_{t d}\right)$ inside the candidate in the green component image,

11. Homogeneity $(H)$ inside the candidate in the green component image,

12. Mean intensity $\left(I_{m_{-} p r}\right)$ of the candidate in the preprocessed green component image,

13. Standard deviation $\left(S_{t d_{P} P r}\right)$ inside the candidate in the preprocessed green component image,

14. Homogeneity $\left(H_{P r}\right)$ of the candidate in the preprocessed green component image.

Fig. 3 illustrates the MAs and HEs detection stages from input color image to the lesion result image overlaid on the original color retinal image.

\section{Results and discussions}

Test operations could be implemented to validate any proposed algorithm by using three criteria, namely image-based classification, lesion-based detection, and pixel-based computation. In this work, 


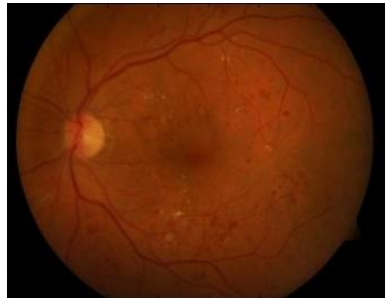

(a)

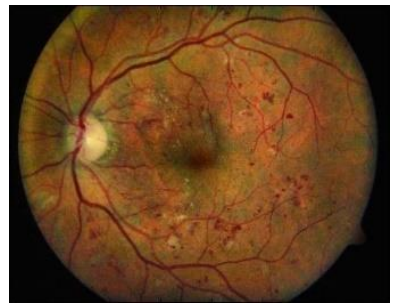

(c)

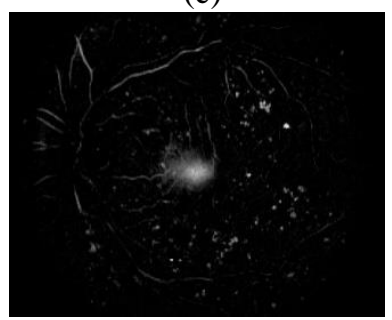

(e)

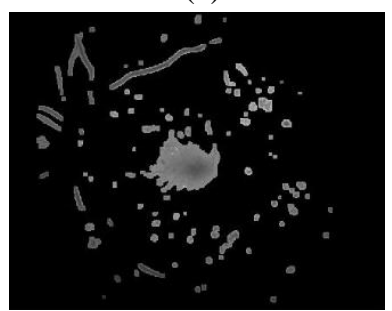

(g)

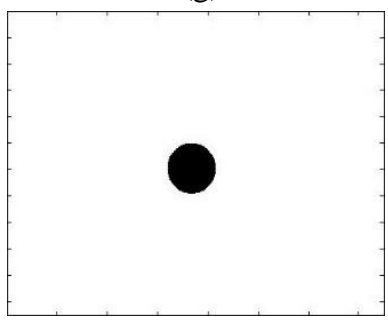

(i)

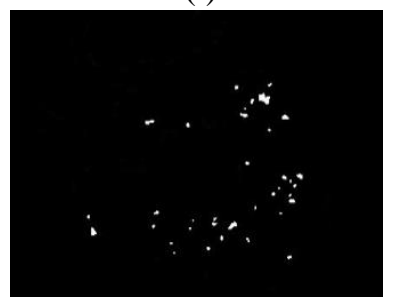

(k)

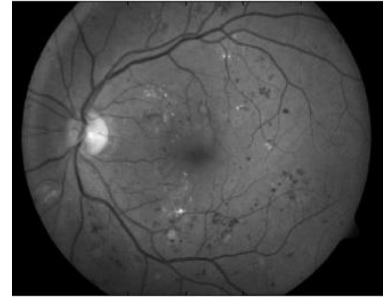

(b)

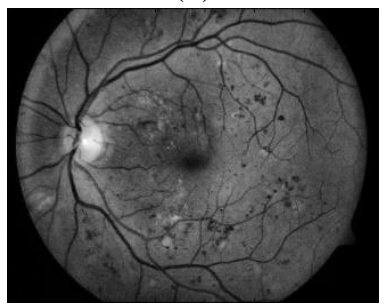

(d)

(f)

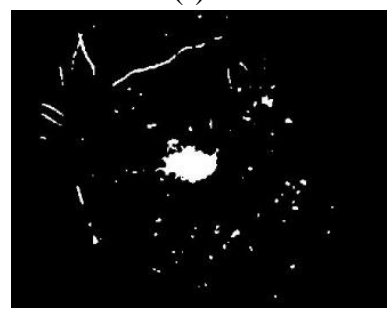

(h)

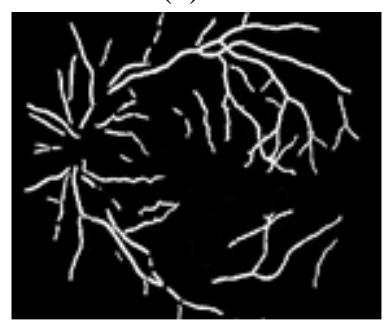

(j)

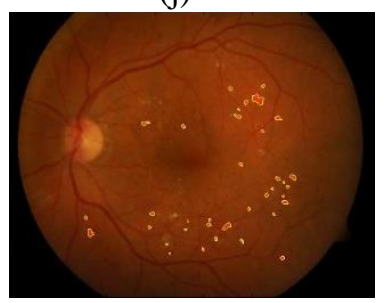

(1)

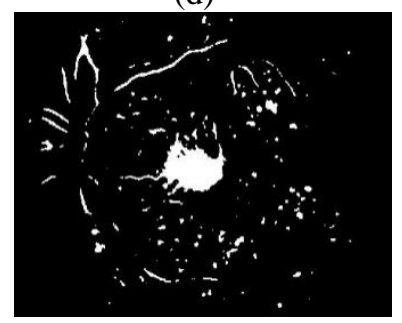

Figure. 3 Steps of MAs and HEs extraction: (a) original color retinal image, (b) green component image, (c) preprocessed color retinal image, (d) preprocessed green component image, (e) preliminary step for MAs and HEs candidates, (f) binary lesion candidates, (g) refined MAs and HEs candidates, (h) binary refined candidates, (i) mask of the fovea, (j) Blood vessels image, $(k)$ final binary result of MAs and HEs image, and (l) binary result overlaid on the original color image several experiments have been carried out to tune the proposed algorithm by using optimal parameters for achieving superior findings. The performance of the proposed method was tested in the three criteria by comparing the results with the specialists annotated images (ground truth).

In pixel-based computations, four different pixel types are calculated: true positives $\left(\mathrm{TP}_{\mathrm{s}}\right)$, false positives $\left(\mathrm{FP}_{\mathrm{s}}\right)$, false negatives $\left(\mathrm{FN}_{\mathrm{s}}\right)$, and true negatives $\left(\mathrm{TN}_{\mathrm{s}}\right)$. Definitions of these pixel types are: -TPs: Number of lesion pixels that are correctly detected as lesions.

-FPs: Number of background pixels that are wrongly detected as lesions.

- $\mathrm{TN}_{\mathrm{s}}$ : Number of background pixels that are correctly identified as background.

-FN $\mathrm{F}_{\mathrm{s}}$ : Number of lesion pixels that are missed (wrongly identified as background).

These quantities are calculated for each individual image to compute performance measures using the following equations (Eqs. 4-7).

$$
\begin{aligned}
& \mathrm{SE}=\mathrm{TP}_{\mathrm{s}} /\left(\mathrm{TP}_{\mathrm{s}}+\mathrm{FN}_{\mathrm{s}}\right) \\
& \mathrm{SP}=\mathrm{TN}_{\mathrm{s}} /\left(\mathrm{TN}_{\mathrm{s}}+\mathrm{FP}_{\mathrm{s}}\right) \\
& \mathrm{PPV}=\mathrm{TP}_{\mathrm{s}} /\left(\mathrm{TP}_{\mathrm{s}}+\mathrm{FP}_{\mathrm{s}}\right) \\
& \mathrm{ACC}=\left(\mathrm{TP}_{\mathrm{s}}+\mathrm{TN}_{\mathrm{s}}\right) /\left(\mathrm{TP}_{\mathrm{s}}+\mathrm{FP}_{\mathrm{s}}+\mathrm{TN}_{\mathrm{s}}+\mathrm{FN}_{\mathrm{s}}\right)
\end{aligned}
$$

The proposed method achieved average pixelbased performance of ACC: $99.3 \%$, SE: $98.8 \%$, SP: 97.7\%, and PPV: $83.7 \%$ using 152 abnormal retinal images.

In lesion-based detection, the four performance measures refer to detection of lesions in terms of segments (regions), while in the image-based classification the success rate (SR) refers to the percentage of abnormal images that are correctly classified as abnormal by the screening method. In lesion-based detection, the proposed method achieved ACC of $99.5 \%$, SE of $99.1 \%$, and SP of 99.3\% using 152 abnormal images. In image-based classification a set of 209 normal and abnormal images were used and achieved a SR of $100 \%$. In the proposed method, the three test criteria i.e. per-pixel basis, per-lesion basis, and per-image basis were performed using publically available databases, namely the DIARETD0 and DIARETD1. The parameters, used in this work, were investigated to manipulate and improve the proposed algorithm performance. Many experiments were carried out to test the influence of parameter changes on the performance measures, namely $\varepsilon$ in the top-down 


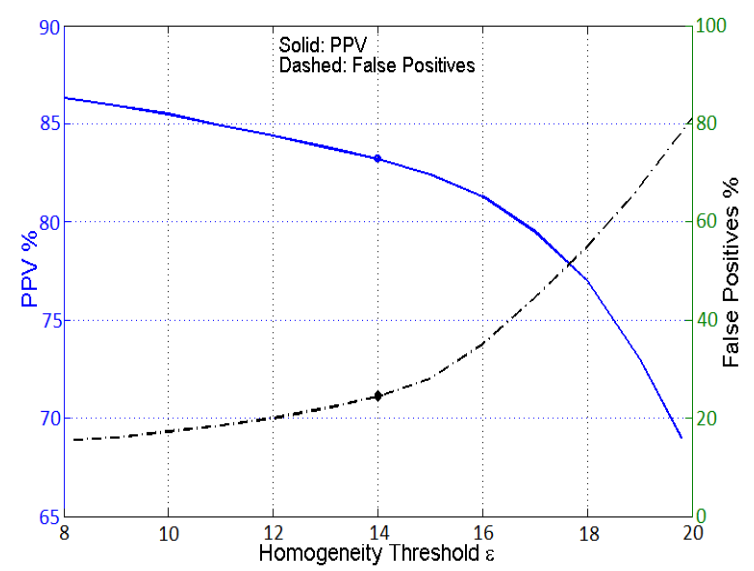

Figure. 4 Influence of the homogeneity threshold $\varepsilon$ on the FPs and PPV rates

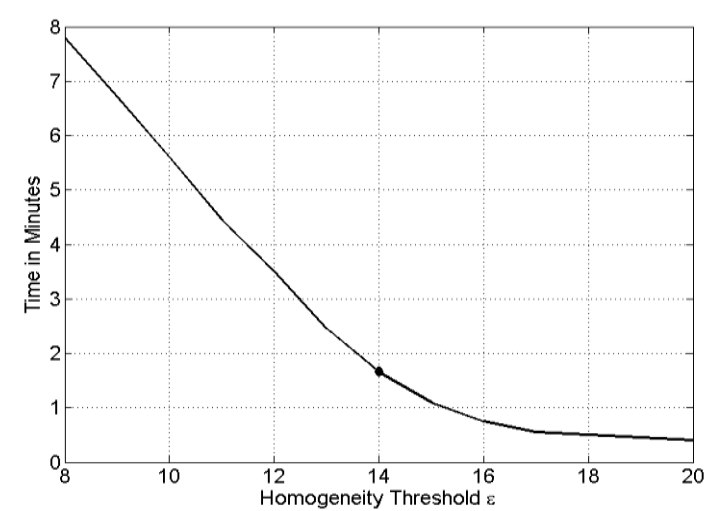

Figure. 5 Influence of the homogeneity threshold $\varepsilon$ on the computation time in the top-down segmentation

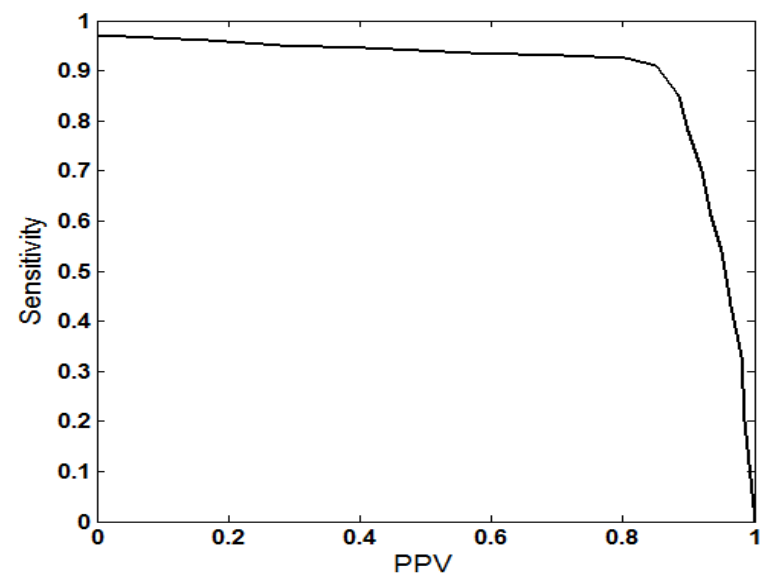

Figure. 6 Influence of the threshold $\alpha$ on the balance between the SE and PPV

segmentation, $\alpha$ in the dynamic thresholding, and $x$ in the region-growing. It appears from a manipulation in the homogeneity threshold $\varepsilon$, it has a noticeable role in compromise between the performance measures and the computational speed. For optimal results with lowest undesired FPs, the ideal value of homogeneity threshold $(\varepsilon)$ is 0 , which means that

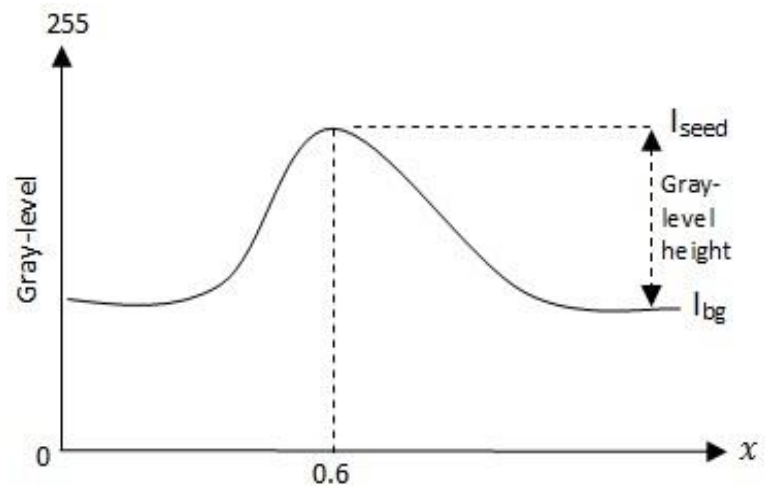

Figure. 7 Intensity profile of MAs and HEs, indicating their gray-level height with respect to the retinal background. $\mathrm{I}_{\text {seed }}, \mathrm{I}_{\mathrm{bg}}$, and $x$ refer to variables in the region-growing criterion

each sub-region has same particular intensity. Practically, the $\varepsilon$ value is subject to the range of pixel intensities in created sub-regions. In this work, all images are normalized to the pixel intensities of unsigned integer 8 (from 0 to 255). Experimental results showed that the good $\varepsilon$ value is 14 (intensity differences are: 0 to 14) in terms of the balance between PPV score and computation time. The $\varepsilon$ of larger than 20 may result in bad performance where the lesion candidates would contain a large number of FPs. From performance measure equations (4-7), it appears that a large number of FPs can affect the PPV dramatically, and also affect ACC and SP slightly in different rates depending on the amount of lesions in that image.

Fig. 4 illustrates the influence of $\varepsilon$ change on the presence rate of FPs and hence on the PPV for $\varepsilon$ range of 8 to 20. Fig 5 illustrates the effect of $\varepsilon$ change on the computation time. In Fig. 4 and Fig. 5 the FPs rates and computation time are both calculated for average of 50 random images, while the PPV represents the average measure of the whole test images (152 images). In the proposed method, the PPV could be improved up to about $87 \%$, by decreasing the $\varepsilon$ to 8 , but this will be at the expense of computation time.

The PPV and SE are both considered as the most informative measures among the others because they are directly related to the detected TPs, where SE measures the proportion of true lesion pixels that are correctly detected, while PPV refers to the proportion of true detected lesion pixels from the whole pixels detected as lesions. The low value of PPV refers to the presence of high value of FPs in the detection results. To improve PPV, the detected lesions have to be further refined where the SE will be certainly affected. Therefore, the compromise between SE and PPV is a big challenge and should be investigated to 
Table 1. Comparison of the proposed method against previous related works (per-pixel basis) using DIARETD0 and/or

\begin{tabular}{|c|c|c|c|c|}
\hline \multicolumn{5}{|c|}{ DIARETD1 } \\
\hline Method & $\mathrm{ACC} \%$ & SE\% & SP\% & PPV\% \\
\hline Wavelet-based Gaussian mixture [8] & 98.3 & 98.9 & 97.7 & -- \\
\hline Boosting, bagging, and Random subspace [10] & 88.2 & 96.1 & 82.1 & -- \\
\hline Convolution neural network [14] & 92 & 84.5 & 94 & 84 \\
\hline Machine learning algorithms [16] & -- & 92.1 & 88.7 & -- \\
\hline Deep neural network [17] & -- & 86.8 & -- & 83.1 \\
\hline Filter banks and hybrid classifier [21] & 99.4 & 98.6 & 99.7 & -- \\
\hline Visual word dictionary-based [22] & -- & 90 & 87 & -- \\
\hline Proposed method & 99.3 & 98.8 & 97.7 & 83.7 \\
\hline
\end{tabular}

Table 2. Comparison of the proposed method against previous related works (per-lesion basis) using DIARETD0 and/or

\begin{tabular}{|lccc|}
\hline Method & DIARETD1 & \\
\hline Mathematical morphology [5] & SE\% & SP\% \\
Dynamic shape features [9] & -- & 100 & 91 \\
Coarse to fine strategy [ 12] & - & 92.9 & 85.9 \\
Maximal stable extremal regions [ 13] & 99.7 & 99.3 & 99.2 \\
Proposed method & -- & 94.9 & 98.9 \\
& 99.5 & 99.1 & 99.3 \\
\hline
\end{tabular}

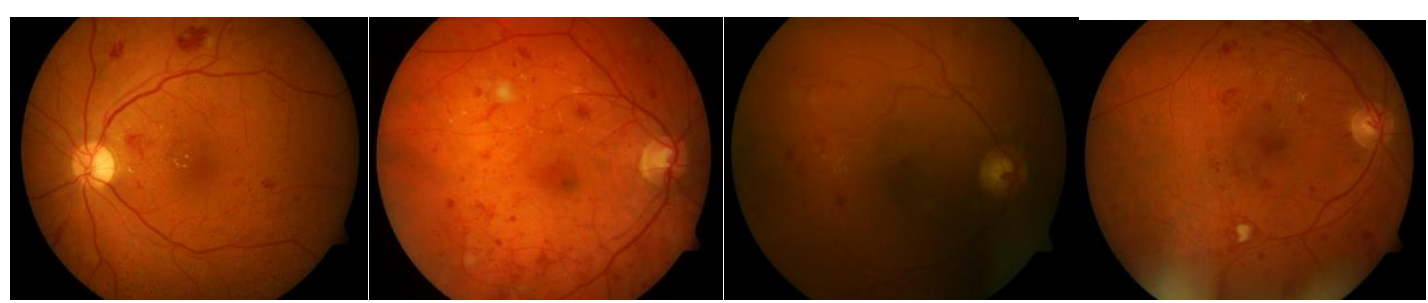

(a)

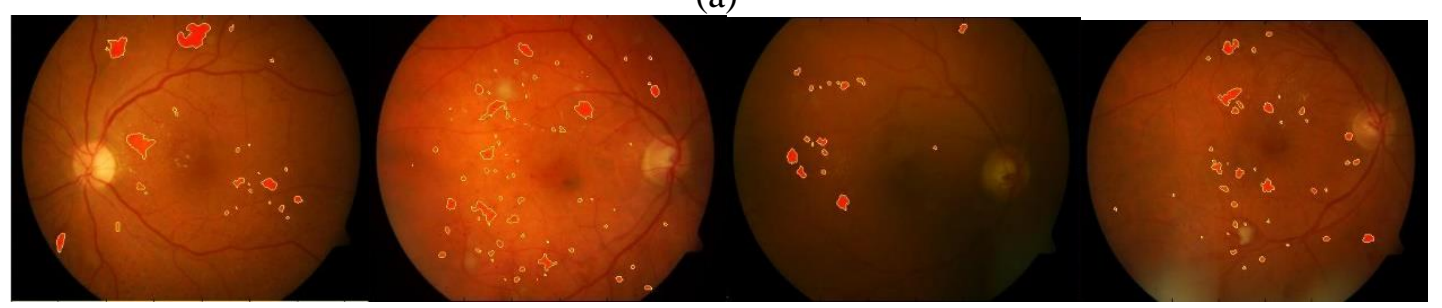

(b)

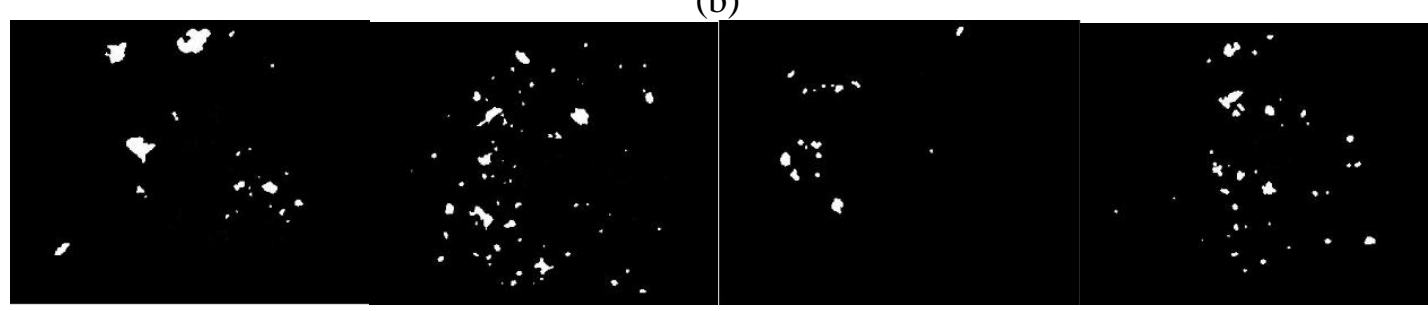

(c)

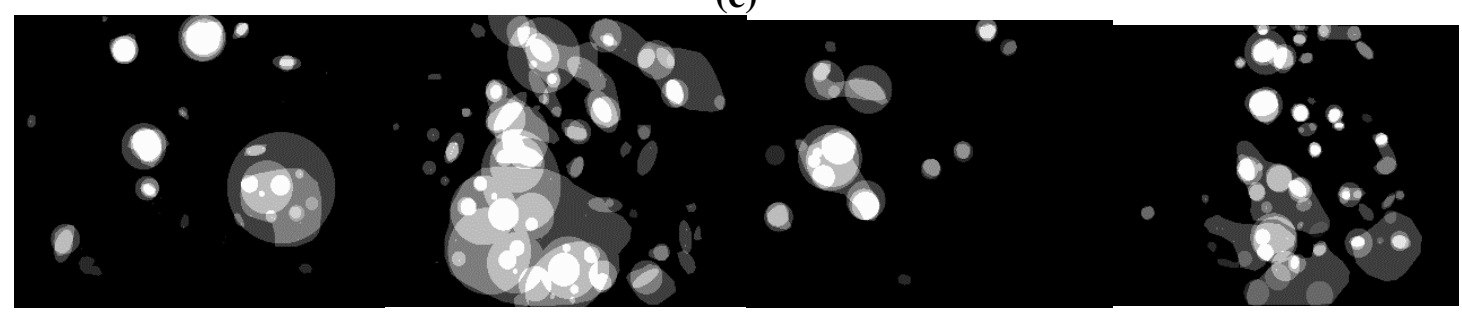

(d)

Figure. 8 Four examples showing the proposed method results and their clinician's ground truth: (a) original color retinal images, (b) binary results superimposed on their corresponding color images, (c) binary results of the proposed method, and (d) clinician's hand-labelled images. 
ensure robust outcomes.

A variation in the threshold level $\alpha$ has noticeable effect on the balance between desired $T P_{s}$ and undesired $\mathrm{FP}_{\mathrm{s}}$ and subsequently on the compromise between SE and PPV as illustrated in Fig. 6. From experimental tests, it appears that changing the $\alpha$ around the selected value $\left(\alpha=0.09 I_{\text {grn }}\right)$ can control the balance between SE and PPV in a way that the increase in $\alpha$ will cause decrease in the SE and increase in the PPV and vice versa. The ability of compromise between performance measures themselves and with computation speed demonstrates that the proposed method response to variations in the main parameters is robust. However, manipulation of these parameters relies on the diagnostic needs and based on the doctor view.

In the region-growing, the factor $x$ assigns how much of MAs and HEs features are required for optimal segmentation. When $x=0.8$, then all features would be included except the lowest $20 \%$, with respect to its height. A unity value of $x$ will cause growing of the objects to exceed the boundaries of the feature and may increase to reach surrounding areas of the retina. To avoid over growing, values of $x$ were selected to be between 0.5 and 0.7 , and this range can lead to satisfactory degree of delineation and will also avoid probability of over growing. A value of $x=$ 0.6 was found to be the optimal for the best performance (see Fig. 7).

Although some real lesions may be missed in the refining and classification steps, a high percentage of MAs and HEs are detected and found to achieve a favorable $\mathrm{SE}$ at lesion level with reasonable computational complexity compared with the other related works. The majority of the missed lesions were connected to the blood vessels and they were masked out as a part of the blood vessels during the refining operations. Conversely, some artifacts may be detected wrongly as MAs or HEs and this is due to presence of small vessels having similar features of MAs or HEs.

The main shortage of the majority of previous related works is that they could not avoid the existence of high undesired FPs along with the high desired TPs and hence high sensitivity. The presence of high FPs affects the measure of PPV and so most of the related works avoid calculating this measure. In medical point of view, both SE and PPV (per-pixel basis) are the most informative performance measures because they together serve to find out severity of lesions precisely. Even for those who tested their algorithm with very good PPV, they calculated it per-lesion basis.
Performance comparison of the proposed method with the previous related works, per-pixel basis, is illustrated in Table 1. The pixel-based quantification of lesions is medically more precise and can present more accurate results than the lesion-based detection. Table 2. illustrates a comparison for performance measures of the proposed method and previous related works per-lesion basis. The comparison in Table 1 prove that the per-pixel performance of the proposed method outperforms the previous related works in terms of SE and PPV together. It appears that most of the related works did not report the PPV to avoid the balance challenge between SE and PPV and then to increase the SE but with a drawback of high FPs. Fig. 8 shows four color images and the proposed method results and their expert annotated images.

\section{Conclusions}

In this work, a new algorithm for the extraction of MAs and HEs from digital fundus images is presented. This method includes segmentation of MAs and HEs candidates using a top-down technique and then an edge detection and applying regiongrowing techniques followed by elimination of blood vessels and fovea traces, as refining operations. A classification operation based on local-region properties was also used to discriminate genuine MAs and HEs from other spurious objects.

Superior performance of MAs and HEs extraction showed that the top-down segmentation is very efficient step in discrimination of homogeneous regions with predefined criteria as a coarse segmentation step. In addition, it has the advantage of high efficiency in discrimination between undesired and linear objects (artifacts) and circular objects i.e. the real MAs and HEs.

Importance of refining the MAs and HEs image from traces of residual blood vessels and the fovea was investigated by many experiments and is found it helps obtain detection results with significantly lower false positives. The refining operations also help reduce computational burden of the classification processes. In the classification and feature selection, it appears that efficient selection of features can significantly help improve the performance of the classifier, but selection of reasonable number of features is also important to avoid a burden of computational complexity.

Anyhow, performance comparison of the proposed method with the previous state of the art methods (in Tables 1 and 2) has showed encouraging results. Based on the ability to discriminate between circular and linear dark objects and its superior SE, 
this method is expected to be preferable for other medical applications for red and dark object detection.

The novelty of our proposed method is in combination of three refining stages and in selecting efficient features in the classification stage. The proposed system could identify MAs and HEs efficiently with average sensitivity of $98.8 \%$ and PPV of $83.7 \%$ which together outperform all the related works per-pixel basis. In addition, the parameters, adopted in this work, are able to manipulate the performance measure rates effectively. The implemented method performance can be further improved by using more features for the MAs and HEs in the classification stage. In future, we intend to develop the current algorithm to achieve a comprehensive system for detecting all types of dark and bright lesions associated with DR.

\section{Conflicts of Interest}

The authors declare no conflict of interest.

\section{Author Contributions}

The conceptualization was from Hussain Jaafar, Hilal Al-Libawy, and Qais Al-Gayem. The methodology was organized by Jaafar, The software, by Jaafar and Al-Libawy. The validation, by Jaafar and Al-Gayem. The formal analysis, by Jaafar and Al-Libawy. The resources, by Al-Gayem. The data curation, by Al-Libawy and Al-Gayem. Writingoriginal draft preparation, by Jaafar. Writingreview and editing, by Al-Libawy. Visualization, by Jaafar, Al-Libawy, and Al-Gayem. Supervision, by Jaafar. project administration, by Jaafar. funding acquisition, by Jaafar, Al-Libawy, and Al-Gayem.

\section{Acknowledgments}

The authors would like to thank the DIARETDB0 Database Centre (Kauppi et al, 2006), the DIARETDB1 Database Centre (Kauppi et al, 2007) and the Centre of Mathematical Morphology, Mines Paris Tech. (Walter et al, 2002) for making their databases publically available. They would also like to thank Dr Ticiana Criddle from the Liverpool Diabetic Eye Screening, Royal Liverpool University Hospital, UK for her kind provision of consultancy and medical expertise.

\section{References}

[1] T. Kauppi, V. Kalesnykiene, J. K. Kamarainen, L. Lensu, I. Sorri, A. Raninen, R. Voutilainen, H. Uusitalo, H. Kalviainen, and J. Pietila, "DIARETDB1: diabetic retinopathy database and evaluation protocol", Technical Report,
Lappeenranta University of Technology, Finland, 2007.

[2] M. Niemeijer, B. Van Ginneken, J. Staal, M. S. Suttorp-Schulten, and M.D. Abràmoff, "Automatic detection of red lesions in digital color fundus photographs", IEEE Transactions on Medical Imaging, Vol. 24, No. 5, pp. 584-592, 2005.

[3] T. Walter, P. Massin, A. Erginay, R. Ordonez, C. Jeulin, and J. C. Klein, "Automatic detection of microaneurysms in color fundus images", Medical Image Analysis, Vol. 11, No. 6, pp. 555566, 2007.

[4] U. R. Acharya, C. M. Lim, E. Y. K. Ng, C. Chee, and T. Tamura, "Computer-based detection of diabetes retinopathy stages using digital fundus images", In: Proc. of the Institution of Mechanical Engineers, Part H: Journal of Engineering in Medicine, Vol. 223, No. 5, pp. 545-553, 2009.

[5] G. B. Kande, T. S. Savithri, and P. V. Subbaiah, "Automatic detection of microaneurysms and hemorrhages in digital fundus images", Journal of Digital Imaging, Vol. 23, No. 4, pp. 430-437, 2010.

[6] S. B. Junior and D. Welfer, "Automatic detection of microaneurysms and hemorrhages in color eye fundus images", International Journal of Computer Science \& Information Technology, Vol. 5, No. 5, pp. 21-37, 2013.

[7] I. Soares, M. Castelo-Branco, and A. M. Pinheiro, "Microaneurysms detection using a novel neighborhood analysis", In: Proc. of the Ophthalmic Medical Image Analysis International Workshop, Iowa Research Online, University of Iowa, 2014.

[8] M. Tamilarasi and K. Duraiswamy, "Automatic detection of microaneurysms using microstructure and wavelet methods", Sadhana, Vol. 40, No. 4, pp. 1185-1203, 2015.

[9] L. Seoud, T. Hurtut, J. Chelbi, F. Cheriet, and J. P. Langlois, "Red lesion detection using dynamic shape features for diabetic retinopathy screening", IEEE Transactions on Medical Imaging, Vol. 35, No. 4, pp. 1116-1126, 2015.

[10] R. F. Ren, P. Cao, W. Li, D. Zhao, and O. Zaiane, "Ensemble based adaptive over-sampling method for imbalanced data learning in computer aided detection of microaneurysm", Computerized Medical Imaging and Graphics, Vol. 55, No. 1, pp. 54-67, 2017.

[11] G. N. Y. Gharaibeh, "A Novel Approach for Detection of Microaneurysms in Diabetic Retinopathy Disease from Retinal Fundus 
Images", Computer and Information Science, Vol. 10, No. 1, pp. 1-5, 2017.

[12] R. T. Mamilla, V. K. R. Ede, and P. R. Bhima, "Extraction of Microaneurysms and Hemorrhages from Digital Retinal Images", $J$. Med. Biol. Eng, Vol. 37, No. 3, pp. 395-408, 2017.

[13] A. Manjaramkar and M. Kokare, "Connected Component Clustering Based Hemorrhage Detection in Color Fundus Images", International Journal of Intelligent Engineering and Systems, Vol. 11, No. 2, pp. 143-151, 2018.

[14] P. Khojasteh, B. Aliahmad, D. K. Kumar, "Fundus images analysis using deep features for detection of exudates, hemorrhages and microaneurysms", BMC Ophthalmol, 18:288, 2018

[15] R. Parmar, R. Lakshmanan, S. Purushotham, and R. Soundrapandiyan, "Detecting Diabetic Retinopathy from Retinal Images Using CUDA Deep Neural Network", Intelligent Pervasive Computing Systems for Smarter Healthcare, pp. 379-396, 2019.

[16] D. Lokuarachchi, L. Muthumal, K. Gunarathna, and T. D. Gamage, "Detection of Red Lesions in Retinal Images Using Image Processing and Machine Learning Techniques", In: Proc. of 2019 IEEE Moratuwa Engineering Research Conf. (MERCon), pp. 550-555, 2019.

[17] L. Zhang, S. Feng, G. Duan, Y. Li, and G. Liu, "Detection of Microaneurysms in Fundus Images Based on an Attention Mechanism", Genes, Vol. 10, No. 10, p p. 817-835, 2019.

[18] T. Walter, J. C. Klein, P. Massin, and A. Erginay, "A contribution of image processing to the diagnosis of diabetic retinopathy-detection of exudates in color fundus images of the human retina", IEEE Transactions on Medical Imaging, Vol. 21, No. 10, pp. 1236-1243, 2002.

[19] T. Kauppi, V. Kalesnykiene, J. K. Kamarainen, L. Lensu, I. Sorri, J. Pietila, H. Kalviainen, and H. Unsitalo, "DIARETDB0: Evaluation database and morphology for diabetic retinopathy algorithm", Technical Report, Lappeenranta University of Tech. and University of Kuopio, Finland, 2006.

[20] H. F. Jaafar, A. K. Nandi, and W. Al-Nuaimy, "Decision support system for the detection and grading of hard exudates from color fundus photographs", Journal of Biomedical Optics, Vol. 16, No. 11, pp. 116001, 2011.

[21] M. U. Akram, S. Khalid, and S. A. Khan, "Identification and classification of microaneurysms for early detection of diabetic retinopathy”, Pattern Recognition, Vol. 46, No. 1, pp. 107-116, 2013.
[22] A. Rocha, T. Carvalho, and H. F. Jelinek, "Points of interest and visual dictionaries for automatic retinal lesion detection", IEEE Transactions on Biomedical engineering, Vol. 59, No. 8, pp. 22442253, 2012. 